\title{
Does magnetically assisted capsule endoscopy improve small bowel capsule endoscopy completion rate? A randomised controlled trial
}

Authors

Institution
Melissa F. Hale, Kaye Drew, Reena Sidhu, Mark E. McAlindon

Academic Unit of Gastroenterology, Sheffield Teaching Hospitals, Sheffield, United Kingdom submitted:

2. July 2015

accepted:

23. November 2015

\section{Bibliography}

DOI http://dx.doi.org/

10.1055/s-0035-1569846

Published online: 0.0 .

Endoscopy International Open

2016; 04: E215-E221

(c) Georg Thieme Verlag KG

Stuttgart · New York

E-ISSN 2196-9736

\section{Corresponding author}

\section{Melissa Hale}

Clinical Research Fellow

Room P39, P Floor

Academic Unit of

Gastroenterology

Royal Hallamshire Hospital

Glossop Road

Sheffield

United Kingdom

Phone: +44 1142712353

Fax: +44 1142712692

Melissa.hale@sth.nhs.uk
Background and study aims: Delayed gastric emptying is a significant factor in incomplete small bowel capsule examinations. Gastric transit could be hastened by external magnetic control of the capsule. We studied the feasibility of this approach to improve capsule endoscopy completion rates.

Patients and methods: Prospective, single-center, randomized controlled trial involving $122 \mathrm{pa}-$ tients attending for small bowel capsule endoscopy using MiroCam Navi. Patients were randomized to either the control group (mobilisation for 30 minutes after capsule ingestion, followed by intramuscular metoclopramide $10 \mathrm{mg}$ if the capsule failed to enter the small bowel) or the intervention group $(1000 \mathrm{~mL}$ of water prior to capsule ingestion, followed by positional change and magnetic steering). Outcome measures were capsule endoscopy completion rate, gastric clarity and distention, relationship of body habitus to capsule endoscopy completion rate (CECR), and patient comfort scores.

\section{Introduction \\ $\nabla$}

Capsule endoscopy is a first-line investigative modality for the small bowel that can be used to obtain circumferential and often detailed mucosal views of the narrow lumen during transit. [1 -4] It is noninvasive, does not require sedation, and is much preferred by patients to intubational endoscopy. [5] Intuitively, however, it seems likely that the volume of the stomach, its collapsed state when fasted, and unusual configuration will hinder the ability of capsule endoscopy to reliably identify pathology proximal to the small bowel. However, we have shown that magnetically-assisted capsule endoscopy (MACE) detects beads sewn into all areas of an ex vivo water-containing porcine stomach as reliably as conventional esophagogastroduodenoscopy, implying that a de-
Results: 122 patients were recruited (61 each to the control and intervention groups: mean age 49 years [range 21-85], 61 females). There was no significant difference in CECR between the two groups $(P=0.39)$. Time to first pyloric image was significantly shorter in the intervention group $(P=0.03)$ but there was no difference in gastric transit times $(P=0.12)$, suggesting that magnetic control hastens capsular transit to the gastric antrum but does not influence duodenal passage. Gastric clarity and distention were significantly better in the intervention group $(P<$ 0.0001 and $P<0.0001$ respectively).

Conclusions: Magnetic steering of a small bowel capsule is unable to overcome pyloric contractions to enhance gastric emptying and improve capsule endoscope completion rate. Excellent mucosal visualisation within the gastric cavity suggests this technique could be harnessed for capsule examination of the stomach.

gree of control may allow a complete examination. [6] Preliminary human studies using magnets to control capsule movement in fluid-filled stomachs also suggest diagnostic yields that compare favorably with esophagogastroduodenoscopy. [7,8] Marelli et al., however, identified gastric pathology using a capsule with a higher frame acquisition rate and imaging devices at either end (Pillcam Eso, Given Imaging, Yoqneam, Israel) without using magnets or other methods of control and with much smaller ingestion volumes. [9] This is consistent with studies of anemic patients having capsule endoscopy primarily to image the small bowel (but providing some gastric images during transit) identifying pathology in the esophagus, stomach and duodenum, missed by esophagogastroduodenoscopy, in up to $10 \%$ of cases. $[10,11]$ These data raise the question as to 


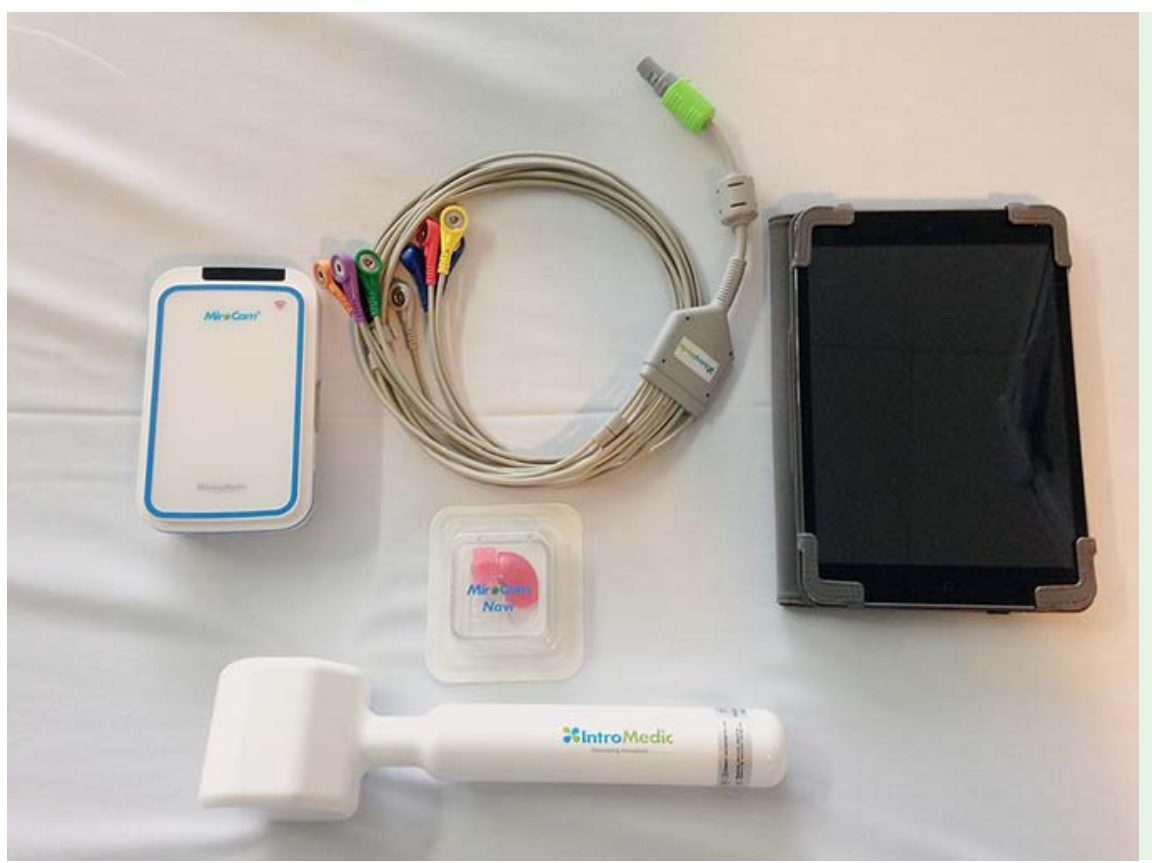

Fig. 1 MiroCam Navi equipment.

whether the degree of control is sufficient to add significantly to the diagnostic potential of this developing technology.

Transit of the capsule through the pylorus is easily recognized and identification of the first duodenal image to mark the beginning of the small bowel is standard practice during capsule endoscopy reporting. By using this landmark, studies suggest that delayed gastric emptying is a major factor in incomplete small bowel capsule endoscopy examinations, which are thought to occur in up to $30 \%$ of small bowel capsule endoscopy procedures. $[12,13]$ The use of prokinetics to stimulate gastric peristalsis has been shown to be effective in reducing gastric transit time and improving CECR. [14,15] These medications carry a risk of adverse reactions, parenteral administration is uncomfortable, and a simple mechanical method that allows sufficient control to steer the capsule into the small bowel may be a desirable alternative.

Understandably there has been an assumption in most trials that volume distension of the stomach using swallowed water will allow visualisation of a greater surface area, act as a mucosal cleansing agent, and provide a suitable medium in which a magnetically controlled capsule can move. Whether it is distension, mucosal cleansing, purging or a combination of these actions, preparation clearly improves mucosal visibility and improves diagnostic yields in small bowel capsule endoscopy. [16-19] Control of capsule movement in a stomach prepared to optimize distension and visibility may pave the way for noninvasive gastric magnetically assisted capsule endoscopy (MACE).

In this study we have compared MACE with a standard protocol to determine if control using a magnet can be demonstrated by the achievement of a readily definable and, in terms of small bowel examination, clinically useful endpoint: small bowel CECR. We have compared two different preparation regimens in terms of clarity of gastric mucosal images and distension to determine if higher volume fluid ingestion improves the quality of gastric visualisation.

\section{Methods}

$\nabla$

\section{Patients}

Patients referred to our institution for small bowel capsule endoscopy between February 2014 and February 2015 were invited to participate. Patients who were younger than age 20, pregnant, unable to speak or understand English or who had permanent pacemakers, intracardiac devices or any other magnetically or electrically controlled devices were excluded. Patient age, sex, height, weight, waist-hip ratio, and body mass index (BMI) were recorded. Small bowel capsule endoscopy was performed after a 12-hour fast and ingestion of $2 \mathrm{~L}$ of polyethylene glycol during the evening prior to the morning examination.

Procedures were performed using MiroCam Navi (Intromedic, Seoul, Korea), a small bowel capsule endoscope containing magnetic inserts (dimensions $24 \times 11 \mathrm{~mm}$, weight $4.2 \mathrm{~g}$, field of view $170^{\circ}$, depth of view $30 \mathrm{~mm}$, operation time 8 hours) ( $\bullet$ Fig. 1 ). Images acquired from one end are captured at a rate of 3 frames per second and transmitted to a data recorder via electric field propagation. These images can be viewed in real-time via a wireless connection to an iPad (Apple Inc., Cupertino, CA) and subsequently after downloading onto a computer workstation. Each participant completed a numeric rating scale questionnaire based on that used by Irvine et al [20] documenting their comfort before and during their procedure, plus a statement as to whether they would be willing to undergo a repeat procedure.

\section{Study design}

This prospective, randomized controlled trial was approved by the local institution and regional ethical review board (NRES Committee Yorkshire \& The Humber 14/YH/1010, Clinical trials number: NCT0228). Patients were randomized using a computer-generated random number sequence to enter one of two protocols:

1. Control protocol

The standard small bowel capsule endoscopy protocol at our institution was used. The capsule was ingested using $200 \mathrm{mLs}$ of water with 5 drops of simethicone. Patients then mobilized 
around the department for 30 minutes. The position of the capsule was established using a real-time viewer. If the capsule failed to enter the duodenum, metoclopramide ( $10 \mathrm{mg}$ intramuscularly) was administered, followed by further mobilization. If the capsule remained in the stomach after a further 30 minutes, an intravenous dose of erythromycin $(250 \mathrm{mg}$ in $100 \mathrm{~mL}$ normal saline) was given.

2. Intervention protocol

A gastric distention volume of $1000 \mathrm{mLs}$ of water containing 5 drops of simethicone was ingested by the patient immediately prior to capsule ingestion. The patient swallowed the capsule with water ad libitum in the sitting position and subsequently adopted the supine position to allow examination to commence. MACE was performed by a single operator (MFH) using a handheld magnet (Navi Controller, Intromedic Ltd). Holding the magnet over the abdominal wall, the capsule could be rotated along its longitudinal axis to obtain close-up images of the gastric mucosa or the lumen by altering the polarity of the magnet with pronation and supination of the wrist. Movement of the magnet in a longitudinal or transverse direction across the abdominal wall was used to achieve subtle movements of the capsule within the dependent pool of water. Larger movements of water were used to wash the capsule from one area to another by positional change of the patient from supine to the right lateral position, occasionally using the left lateral position if orientation of the capsule within the stomach was proving difficult.

Once enough imaging had been acquired to allow orientation within the stomach, the maneuvers described were used to try and drag the capsule into the antrum and orientate it toward the pylorus. Thereafter, a variety of approaches were used to assist transpyloric passage, most commonly involving the patient leaning toward the right lateral position and the magnet held over the right lateral chest wall or posteriorly as far as the vertebral column. If the capsule failed to pass through the pylorus using magnetic control, within the allotted time limit of 30 minutes, the patient transferred to the relevant section of the control protocol.

\section{Analysis}

Based on the available literature and our local data, assuming a CECR of $70 \%$ using our standard (control) protocol, 60 patients were required per group to be able to detect a $20 \%$ improvement in small bowel (CECR) with a $5 \%$ two-sided significance level, $80 \%$ power. Quantitative data are summarized with parametric statistics, the mean and standard deviation or with non-parametric statistics, the median and interquartile range. The unpaired $t$ test was used to compare age between the two study groups and the $\mathrm{X}^{2}$ test was used to compare sex and indication for small bowel capsule endoscopy between study groups. First pyloric view and gastric transit time were not normally distributed and thus the Mann Whitney test was used to compare the differences in these variables between the two groups. Gastric distention and mucosal visualisation (as measured on a 1-4 scale) between the two groups was also compared using a Mann Whitney test, although we must highlight there are some limitations of using this test when there are only four possible outcomes to compare. Small bowel transit time between the two groups was compared using an unpaired $t$ test. Linear regression was used to assess the correlation between BMI or waist-hip ratio and gastric transit time. An unpaired $t$ test was used to assess the correlation be- tween BMI or waist-hip ratio and CECR. The diagnostic yield, CECR and patient willingness to undergo a repeat procedure were measured as a simple "yes" or "no" and thus were examined using Fisher's exact test.

The small bowel capsule endoscopy videos were read and reported in the conventional manner, which included annotation of the anatomical landmarks (first gastric, duodenal and cecal images), by one of two experienced capsule endoscopists (K.D, M.E.M). One individual (M.E.M) studied all gastric imaging, assigned a score (adapted from that used by Eliakim et al. 1: excellent; 2: good; 3: fair; 4: poor) [21] for both clarity of the gastric mucosa images and distension of the gastric lumen and also marked the first image seen during the examination of the pylorus. All reviews were done in random order and blinded to the protocol assigned.

\section{Outcome measures}

The primary outcome measure for the effect of magnetic control on transpyloric transit of the capsule was CECR. Secondary outcome measures were gastric mucosal clarity, gastric distension, relationship of body habitus to CECR, and patient comfort scores.

\section{Results}

\section{$\nabla$}

A total of 122 patients were prospectively recruited to the study between February 2014 and February 2015. The capsule was retained in the stomach for the duration of the procedure in two patients, thus only demographic data and CECR were analysed ( $\bullet$ Fig.2). Eleven patients did not return their comfort questionnaires and thus were excluded from the patient tolerance analysis. No significant adverse events were recorded.

\section{Demographic data and indications for small bowel capsule endoscopy}

Patient demographics and indications for small bowel capsule endoscopy are shown in $\bullet$ Table 1. Demographic data and CECR from 122 patients and full data from 120 patients were submitted for final analysis (34 men, mean (SD) age $49.6 \pm 17.8$ years, range 20-85 years). There was no significant difference in age $(P=0.86)$, sex $(P=0.55)$, indication for small bowel capsule endos-

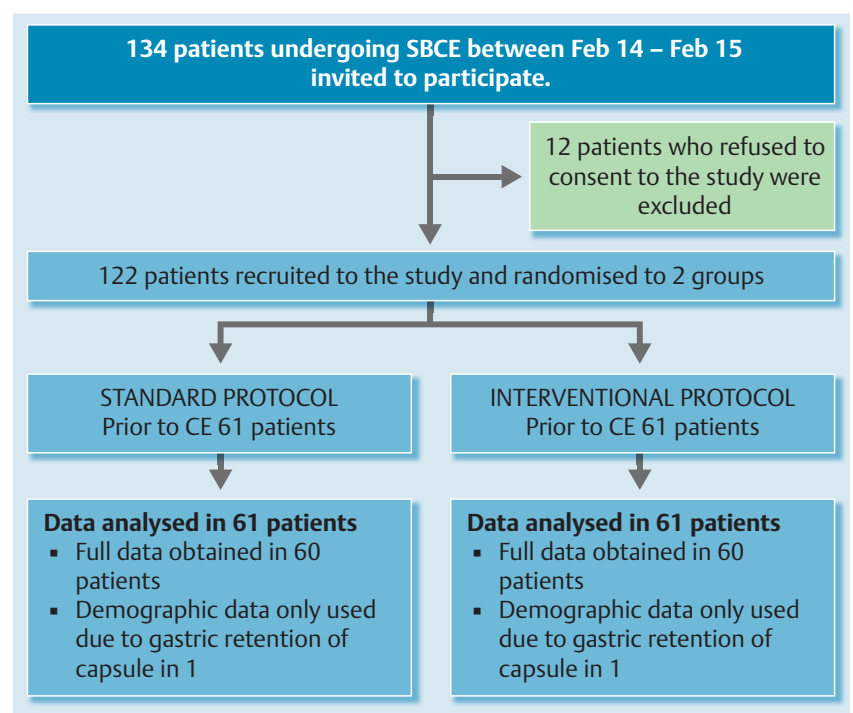

Fig. 2 Study flow diagram. 
copy $(P=0.58)$, BMI $(P=0.37)$ or waist-hip ratio $(P=0.53)$ between the two groups ( $\odot$ Table1.) All but one patient (in the intervention group) were ambulatory outpatients at the time of the procedure.

\section{Transit times, CECRs and adverse events}

The overall CECR for all study patients was $87.6 \%$, with a median gastric transit time of 35.5 minutes (12.5-65.0) and mean small bowel transit time of $276.8( \pm 132)$ minutes. No significant difference in esophageal transit time, CECR or small bowel transit time was noted between the two groups $(P=0.54, P=0.39$ and $P=0.42$ respectively). However, the pylorus was visualized in a significantly shorter time in the intervention group $(P=0.03)$, but this did not impact pyloric transit because there was no significant difference in overall gastric transit time between the two groups $(P=0.12)$ ( $\odot$ Table 2). Gastric mucosal clarity and distention were graded significantly better in the intervention group based on the $1-4$ scale used $(P<0.0001$ and $P<0.0001$ respectively), these data are further illustrated in $\odot$ Table 3.

Subanalysis of the intervention group showed that in 23 (37.7\%) procedures, the capsule could be manipulated into the duodenum under magnetic control within the 30-minute timeframe. There was no significant association between BMI and CECR $(P=$ $0.51)$ or waist-hip ratio and CECR $(P=0.94)$. Similarly, there was no significant association between BMI and gastric transit time, assessed using linear regression, $\left(R_{2} 0.002\right)$ or waist-hip ratio and gastric transit time $\left(R_{2}\right.$ 5.987). There were no known cases of capsule retention or serious adverse events in any of the study participants.

\section{Magnetic steering and gastric visibility}

After ingestion, the capsule was commonly propelled to a dependent area along the greater curvature. Manipulation of the external magnet at the level of the xiphisternum with the patient in the supine position could affect tilting and rotational movements of the capsule in order to determine the location of the capsule and the direction of the gastric antrum ( $\bullet$ Fig.3) Right lateral movement of the magnetic controller could direct the capsule to the gastric antrum in some cases, whereas a change to the right lateral position was required in some participants. Once the pylorus was visualized, further movements with the external magnet over the epigastrium, right upper quadrant, and back (depending on body habitus) could bring the pylorus directly in view. Manipulation of the capsule depended on a complex assessment of the participant's body habitus, degree of gastric distention and likely surface anatomy of the stomach in varying positions, together with fine movements of the external magnet and interpretation of images received to the iPad viewer.

\section{Patient tolerance}

Patients completed a numeric rating scale questionnaire before the procedure documenting their current and anticipated procedural pain and discomfort on a $1-10$ scale $(0=$ no pain/discomfort, $10=$ worst pain/discomfort ever). After the procedure they were required to document their actual pain, discomfort, and distress during the procedure on the same $1-10$ scale. Finally each participant was asked whether he or she would consider undergoing a repeat procedure. There were no significant differences in procedural pain, discomfort or distress between the two groups (৫ Table 4). $98 \%$ of participants in each group were willing to undergo a repeat procedure if necessary.
Table 1 Patient demographics and indications for small bowel capsule endoscopy.

\begin{tabular}{|c|c|c|}
\hline & \multicolumn{2}{|l|}{ Group } \\
\hline & Control & Intervenion \\
\hline Number & 61 & 61 \\
\hline Mean (SD) age, years & $49.5 \pm 18.1$ & $49.5 \pm 17.8$ \\
\hline \multicolumn{3}{|l|}{ Sex } \\
\hline Men & 15 & 19 \\
\hline Women & 45 & 42 \\
\hline \multicolumn{3}{|l|}{ Indication for CE (number (\%)) } \\
\hline OGIB or IDA & $11(18)$ & $14(23)$ \\
\hline Abdominal pain and/or diarrhoea & $31(52)$ & $26(43)$ \\
\hline Known Crohn's disease & $6(10)$ & $13(21)$ \\
\hline Coeliac disease & $9(15)$ & $7(11)$ \\
\hline Polyposis syndromes & $1(2)$ & $0(0)$ \\
\hline Other & $2(3)$ & $1(2)$ \\
\hline Mean BMI (SD) & $29 \pm 9.5$ & $27 \pm 5.4$ \\
\hline Mean Waist-Hip Ratio (SD) & $0.89 \pm 0.15$ & $0.90 \pm 0.08$ \\
\hline
\end{tabular}

SD, standard deviation; CE, capsule endoscopy; OGIB, obscure gastrointestinal bleeding; IDA, iron deficiency anemia; BMI, body mass index

Table 2 Transit times, CECRs and adverse events.

\begin{tabular}{|c|c|c|c|}
\hline & \multicolumn{3}{|l|}{ Group } \\
\hline & Control & Intervention & $P$ value \\
\hline Median OTT (IQR) (sec) & $19(10-34)$ & $19(13-25)$ & 0.54 \\
\hline $\begin{array}{l}\text { Median first pyloric } \\
\text { image (IQR) (min) }\end{array}$ & $8(3-25)$ & $5(3-10)$ & 0.03 \\
\hline $\begin{array}{l}\text { Median GTT (IQR) } \\
\text { (min) }\end{array}$ & $23(13-66)$ & $55(13-64)$ & 0.12 \\
\hline Mean SBTT (SD) (min) & $327( \pm 127)$ & $317( \pm 128)$ & 0.42 \\
\hline CECR (\%) & 87 & 89 & 0.39 \\
\hline $\begin{array}{l}\text { Gastric mucosal clarity } \\
\text { (1-4 scale) }\end{array}$ & $3(2-3)$ & $1(1-2)$ & $<0.0001$ \\
\hline $\begin{array}{l}\text { Gastric distention } \\
\text { (1-4 scale) }\end{array}$ & $3(3-4)$ & $2(1-3)$ & $<0.0001$ \\
\hline Diagnostic yield (\%) & 31 & 36 & 0.70 \\
\hline
\end{tabular}

OTT, esophageal transit time; IQR, interquartile range; sec, seconds; min, minutes; GTT, gastric transit time; SBTT, small bowel transit time; CECR, capsule endoscopy completion rate

Table 3 Grading of gastric mucosal clarity and distension.

\begin{tabular}{|c|c|c|c|}
\hline & & \multicolumn{2}{|l|}{ No. of patients } \\
\hline & & Control Group & $\begin{array}{l}\text { Intervention } \\
\text { Group }\end{array}$ \\
\hline \multirow{4}{*}{$\begin{array}{l}\text { Gastric mucosal } \\
\text { clarity Score }(1-4)\end{array}$} & 1 & 14 & 33 \\
\hline & 2 & 16 & 23 \\
\hline & 3 & 20 & 3 \\
\hline & 4 & 11 & 2 \\
\hline \multirow{4}{*}{$\begin{array}{l}\text { Gastric distension } \\
\text { score }(1-4)\end{array}$} & 1 & 6 & 28 \\
\hline & 2 & 6 & 15 \\
\hline & 3 & 33 & 15 \\
\hline & 4 & 16 & 3 \\
\hline
\end{tabular}




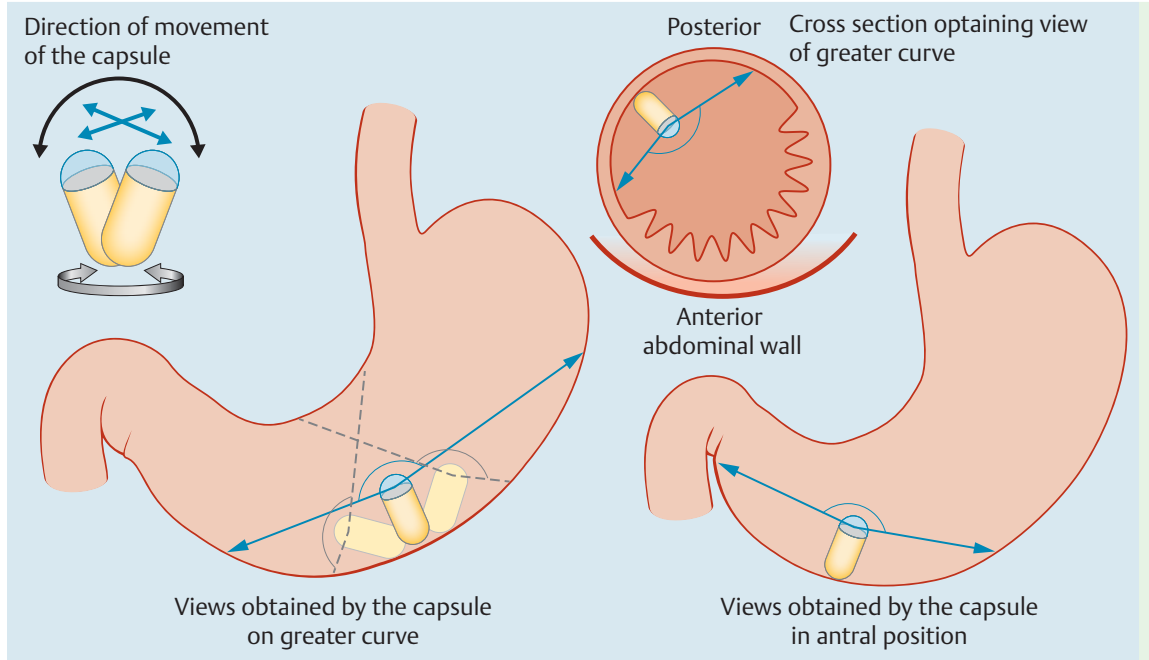

Fig. 3 Schematic of MACE maneuvers.

\begin{tabular}{|c|c|c|c|}
\hline & \multicolumn{3}{|l|}{ Group } \\
\hline & Control & Intervention & $P$ value \\
\hline $\begin{array}{l}\text { Pre-procedural discomfort } \\
\text { median (IQR) }\end{array}$ & $1(1-3)$ & $1(0-3)$ & 0.90 \\
\hline $\begin{array}{l}\text { Pre-procedural pain } \\
\text { median (IQR) }\end{array}$ & $0(0-2.8)$ & $0(0-3)$ & 0.98 \\
\hline $\begin{array}{l}\text { Expected discomfort } \\
\text { median (IQR) }\end{array}$ & $2(0-4)$ & $2(0-3.8)$ & 0.98 \\
\hline $\begin{array}{l}\text { Expected pain } \\
\text { median (IQR) }\end{array}$ & $2(0-4)$ & $1(0-3)$ & 0.42 \\
\hline $\begin{array}{l}\text { Procedural pain } \\
\text { median (IQR) }\end{array}$ & $0(-)$ & $0(-)$ & 0.58 \\
\hline $\begin{array}{l}\text { Procedural discomfort } \\
\text { median (IQR) }\end{array}$ & $0(0-1)$ & $0(-)$ & 0.43 \\
\hline $\begin{array}{l}\text { Procedural distress } \\
\text { median (IQR) }\end{array}$ & $0(-)$ & $0(-)$ & 0.54 \\
\hline Repeat procedure (\%) ${ }^{*}$ & 98 & 98 & \\
\hline
\end{tabular}

Table4 Patient tolerance.

IQR, interquartile range

*Willingness to undergo a repeat procedure.

\section{Discussion}

$\nabla$

Magnetic control does not appear to be clinically useful in improving CECR as a tool to assist in small bowel capsule endoscopy and this was not affected by body habitus. However, the more rapid identification of the pylorus suggests that a magnet can exert a degree of control that allows endoscopist orientation in the stomach and which is likely to be clinically useful for gastric capsule endoscopy. Gastric preparation with a liter of ingested water containing simethicone offers better gastric mucosal clarity and distension than a $200-\mathrm{mL}$ volume. It follows that a clean, adequately distended stomach is an important aspect of successful MACE, allowing the operator to identify gastric landmarks more easily and thus manipulate the capsule more effectively to achieve the required end. Importantly, MACE is a procedure that induces no measurable pain, discomfort or distress in patients. Further studies of control and preparation are needed to understand the potential of MACE as a noninvasive upper gastrointestinal diagnostic tool.

After ingestion, the capsule commonly dropped to the dependent part of the distal gastric body and could be manipulated to view both the antropyloric region and upper gastric body simultaneously. As with the study by Rey et al. using the Siemens elec- tromagnetic guidance system, the pylorus was readily identified and while progress could be made towards it, it could not be done sufficiently quickly to avoid posterior displacement by powerful antropyloric contractions. [7] Efforts to do so were further hampered by the need to place the magnet behind the patient to attempt to pull the capsule along the superoposterior axis in which the antroduodenum tends to lie. [22] Therefore as a tool to enhance GTT to optimize small bowel examination, it does not appear to be better than a standard protocol using prokinetic agents. For the purposes of gastric examination, however, retention of the capsule is desirable and the antropyloric region, a common site for pathology, was well visualized ( $\bullet$ Fig.4). Furthermore, peristaltic contractions could, of course, be attenuated using anticholinergic agents such as hyoscine $[23,24]$. Whether a combination of a promotility agent and magnetic steering could be used together to enhance pyloric transit of the capsule would require further research.

Strength of attraction diminishes exponentially with increasing distance between magnets and several studies have suggested that this might hamper MACE in obese patients. [22,25,26] However, because magnetic control failed to offer any advantage over a standard protocol when using CECR as the main outcome measure, it seems unlikely that this question has been adequately ad- 


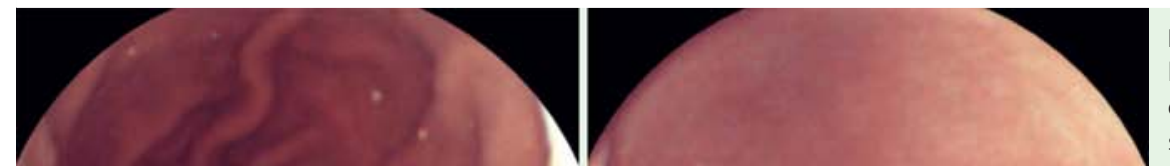

Fig. 4 a Longitudinal view of the gastric body and lesser curve. b Gastric antrum. c Pre-pyloric erosion. d Angioectasia in the cardia. e NSAID-related erosive gastropathy. f Fundic gland polyps. g Pancreatic rest.
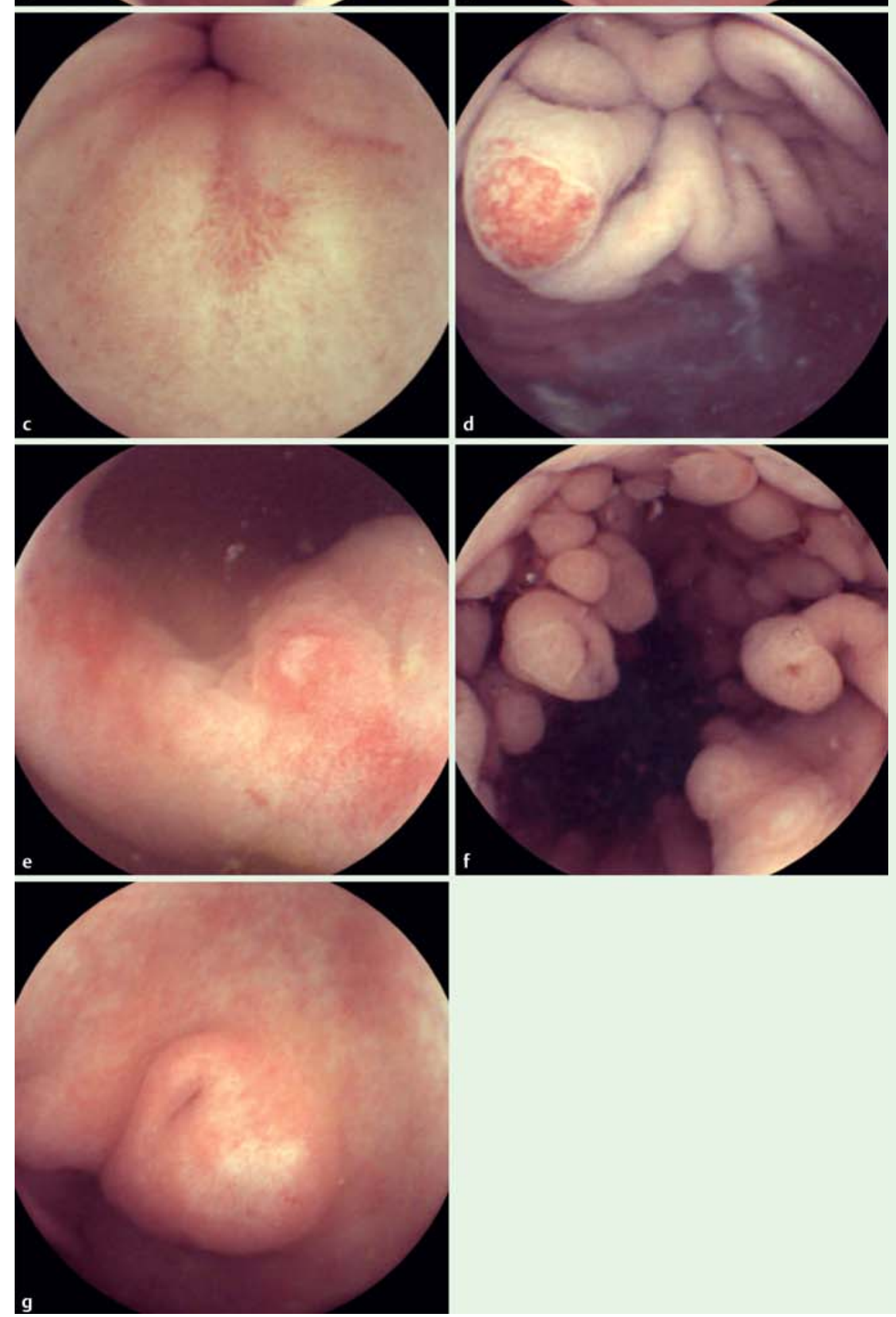
dressed and further study is needed using different markers of control.

Inflation of the stomach during esophagogastroduodenoscopy allows the endoscopist to distend the stomach and flatten rugal folds, maximizing the chances of visualising the whole gastric mucosal surface, therefore minimizing the risk of missed pathology. Such a facility is not an option in a remote technology like capsule endoscopy, but may be afforded indirectly by the ingestion of water or gas-producing substances. Our study shows that ingestion of a liter of water significantly improves gastric mucosal clarity and distension compared to a $200-\mathrm{mL}$ volume. Other studies have empirically used water volumes of $300 \mathrm{~mL}$ [9] and $1300 \mathrm{~mL}$ (in three divided doses over 75 minutes) [7,27] alone, or $500 \mathrm{~mL}$ [25] and $1000 \mathrm{~mL}$ [26] with gas-producing substances. The addition of simethicone as an anti-foaming agent may have contributed to opacity of the gastric content in other studies $[25,26]$ but appears to have been used in larger doses than in our protocol. Further investigation is needed to determine the optimal preparation, but the evidence from this study is that higher ingestion volumes improve the distension and visibility of the gastric mucosa and, thus, the overall quality of the examination.

The Siemens guidance system generates a magnetic field of varying strength and capsule movement is controlled using joysticks. It may, therefore, allow more subtle control than is afforded by a handheld magnet, which is reliant on the operator adjusting the distance of the magnet from, and location over, the skin surface. The magnet weighs $1.2 \mathrm{~kg}$, so subtlety of control probably diminishes as the arm fatigues. However, endoscopists have long been used to learning complex procedures that require high levels of manual dexterity and the attraction of a handheld magnet is simplicity of operation, portability and comparatively minimal cost.

\section{Conclusions}

$\nabla$

Manipulation of a capsule endoscope in the stomach using magnetic steering is feasible but it does not overcome pyloric contractions to enhance gastric emptying or improve CECR. Improved gastric mucosal clarity and distension are observed using higher volumes of ingested water and the addition of simethicone. Further work is recommended to explore the potential of this novel technology.

\section{Competing interests: None.}

\section{References}

1 Triester SL, Leighton JA, Leontiadis GI et al. A meta-analysis of the yield of capsule endoscopy compared to other diagnostic modalities in patients with obscure gastrointestinal bleeding. Am J Gastroenterol 2005; 100: 2407 - 2418

2 Triester SL, Leighton JA, Leontiadis GI et al. A meta-analysis of the yield of capsule endoscopy compared to other diagnostic modalities in patients with non-stricturing small bowel Crohn's disease. Am J Gastroenterol 2006; 101: 954-964

3 Rokkas T, Niv Y. The role of video capsule endoscopy in the diagnosis of celiac disease: a meta-analysis. Eur J Gastroen Hepat 2012; 24: 303 308

4 Sidhu R, McAlindon ME. The use of capsule endoscopy for the investigation of small bowel tumors: experience from a United Kingdom single center. Digest Dis Sci 2011; 56: 2763
5 Irvine A. Assessing Tolerability of Endoscopy. University of Sheffield 2013

6 Hale MF, Rahman I, Drew K et al. Magnetically steerable gastric capsule endoscopy is equivalent to flexible endoscopy in the detection of markers in an excised porcine stomach model: results of a randomized trial. Endoscopy 2015; 47: 650-653

7 Rey JF, Ogata $H$, Hosoe $N$ et al. Blinded nonrandomized comparative study of gastric examination with a magnetically guided capsule endoscope and standard videoendoscope. Gastrointest Endosc 2012; 75: $373-381$

8 Denzer UW, Rosch T, Hoytat $B$ et al. Magnetically guided capsule versus conventional gastroscopy for upper abdominal complaints: a prospective blinded study. J Clin Gastroenterol 2015; 49: 101 - 107

9 Marelli LJF, Jackson L, Palmer $H$ et al. A pilot study comparing ESO-2 capsule endoscopy with conventional upper endoscopy for the assessment of uncomplicated heartburn and dyspepsia. Frontline Gastroenterol 2013; 4: 96 - 101

10 Sidhu R, Sanders DS, Kapur K et al. Capsule endoscopy changes patient management in routine clinical practice. Digest Dis Sci 2007; 52: $1382-1386$

11 Kitiyakara T, Selby $W$. Non-small-bowel lesions detected by capsule endoscopy in patients with obscure GI bleeding. Gastrointest Endosc 2005; 62: 234-238

12 Liao Z, Gao R, Xu C et al. Indications and detection, completion, and retention rates of small-bowel capsule endoscopy: a systematic review. Gastrointest Endosc 2010; 71: 280-286

13 Lee MM, Jacques A, Lam E et al. Factors associated with incomplete small bowel capsule endoscopy studies. World J Gastroentero 2010; 16: $5329-5333$

14 Sidhu R, Drew K, Sanders DS et al. Does the selective use of metoclopramide improve the completion rate of small-bowel capsule endoscopy? Gastrointest Endosc 2010; 72: 670-671

15 Koulaouzidis A, Dimitriadis S, Douglas $S$ et al. The Use of domperidone increases the completion rate of small bowel capsule endoscopy: does this come at the expense of diagnostic yield? J Clin Gastroenterol 2014; 49: 395-400

16 Kotwal VS, Attar BM, Gupta S et al. Should bowel preparation, antifoaming agents, or prokinetics be used before video capsule endoscopy? A systematic review and meta-analysis Eur J Gastroen Hepat 2014; 26: 137-145

17 Song HJ, Moon JS, Do JH et al. Guidelines for Bowel Preparation before Video Capsule Endoscopy. Clin Endosc 2013; 46: 147-154

18 Koulaouzidis A, Giannakou A, Yung DE et al. Do prokinetics influence the completion rate in small-bowel capsule endoscopy? A systematic review and meta-analysis Curr Med Res Opin 2013; 29: 1171 - 1185

19 Wu L, Cao Y, Liao C et al. Systematic review and meta-analysis of randomized controlled trials of Simethicone for gastrointestinal endoscopic visibility. Scand J Gastroentero 2011; 46: 227-235

20 Irvine AJ SD, Hopper A, Kurien $M$ et al. How does tolerability of double balloon enteroscopy compare to other forms of endoscopy? Frontline Gastroenterol 2015: DOI 10.1136/flgastro-2014-100550

21 Eliakim R, Yassin K, Niv $Y$ et al. Prospective multicenter performance evaluation of the second-generation colon capsule compared with colonoscopy. Endoscopy 2009; 41: 1026-1031

22 Rahman I, Kay M, Bryant $T$ et al. Optimizing the performance of magnetic-assisted capsule endoscopy of the upper GI tract using multiplanar CT modelling. Eur J Gastroen Hepat 2015; 27: 460 - 466

23 Petrillo $M$, Lazzaroni $M$, Saponati $G$ et al. The use of rociverine as premedication for endoscopy of the upper gastro-intestinal tract: a double-blind controlled clinical trial. Curr Med Res Opin 1980; 7: 73-76

24 Hiki N, Kurosaka H, Tatsutomi $Y$ et al. Peppermint oil reduces gastric spasm during upper endoscopy: a randomized, double-blind, doubledummy controlled trial. Gastrointest Endosc 2003; 57: 475-482

25 Keller J, Fibbe $C$, Volke $F$ et al. Inspection of the human stomach using remote-controlled capsule endoscopy: a feasibility study in healthy volunteers (with videos). Gastrointest Endosc 2011; 73: 22-28

26 Liao Z, Duan XD, Xin L et al. Feasibility and safety of magnetic-controlled capsule endoscopy system in examination of human stomach: a pilot study in healthy volunteers. J Interv Gastroenterol 2012; 2: $155-160$

27 Rey JF, Ogata H, Hosoe $N$ et al. Feasibility of stomach exploration with a guided capsule endoscope. Endoscopy 2010; 42: 541 -545 